\title{
Descriptive study of an outbreak of equine sarcoid in a population of Cape mountain zebra (Equus zebra zebra) in the Gariep Nature Reserve
}

\author{
$P J \mathrm{Nel}^{\mathrm{a}^{*}}, \mathrm{H}$ Bertschinger ${ }^{\mathrm{b}}, \mathrm{J} \mathrm{Williams}^{\mathrm{c}}$ and $\mathrm{P} N$ Thompson $^{\mathrm{b}}$
}

\begin{abstract}
An outbreak of equine sarcoid occurred in a population of Cape mountain zebra (Equus zebra zebra) at the Gariep Nature Reserve located in the southern Free State Province of South Africa in 1996. The course of the outbreak during 1996 to 2003 is described. During this period the average population size was 69 animals. Initially (1996) all affected animals were removed from the population. New cases continued to manifest and the incidence varied between $4.6 \%$ and $17.6 \%$. Prevalence reached $24.7 \%$ in 2002 . No sexual predilection was noticed in the 39 recorded cases. Of the affected individuals, $64 \%$ had a single lesion and no animal had more than 4 lesions. In males, the majority of lesions occurred in the inguinal area $(55.17 \%)$, whereas in females they mostly occurred on the head and neck $(41.38 \%)$. Lesions can increase $260 \%$ in size annually and may impede movement.

Key words: Cape mountain zebra, equine sarcoid, Gariep Nature Reserve, tumour.

Nel P J, Bertschinger H, Williams J, Thompson P N Descriptive study of an outbreak of equine sarcoid in a population of Cape mountain zebra (Equus zebra zebra) in the Gariep Nature Reserve. Journal of the South African Veterinary Association (2006) 77(4): 184-190 (En.). Free State Department of Tourism, Environmental and Economic Affairs, Private Bag X20801, Bloemfontein, 9300 South Africa.
\end{abstract}

\section{INTRODUCTION}

Sarcoids were first described and characterised as a clinical entity in the equine species in South Africa by Jackson in $1936^{7}$. Equine sarcoid was found in different studies to be the most common skin neoplasm in horses, mules and donkeys $^{3,7,12}$. The World Health Organizsation (WHO) Tumour Classification System lists the sarcoid as a benign tumour and although it can be locally invasive, it is strictly limited to the skin and directly-underlying tissues and does not spread to internal organs ${ }^{9}$. There is considerable evidence that equine sarcoid is caused by a virus closely related to or identical to bovine papilloma virus $(\mathrm{BPV})^{12}$. Intra-dermal inoculation of horses with cell-free extracts from bovine skin tumours containing BPV has been shown to cause lesions resembling equine sarcoids $^{12}$. In a study of histologically confirmed sarcoids from 58 horses, DNA for BPV-1 and BPV- $2^{18}$ could be amplified from every sample.

${ }^{\text {a}}$ Free State Department of Tourism, Environmental and Economic Affairs, Private Bag X20801, Bloemfontein, 9300 South Africa.

${ }^{b}$ Department of Production Animals, Faculty of Veterinary Science, Private Bag X04, Onderstepoort, 0110 South Africa.

${ }^{\circ}$ Department of Para-clinical Studies - Pathology Section, Faculty of Veterinary Science, Private Bag X04, Onderstepoort, 0110 South Africa.

*Author for correspondence. E-mail: nelpj@absamail.co.za Received: August 2006. Accepted: November 2006.
The Cape mountain zebra (CMZ, Equus zebra zebra) is described as a subspecies of Equus zebra; the mountain zebra ${ }^{14}$. The species is vulnerable ${ }^{6}$ and occurs as a number of isolated populations, 1 of which is in the Gariep Nature Reserve (GNR).

Sarcoids were first observed in the CMZ of GNR in June 1995 when nature conservators reported 'large growths' on some animals. In January 1996, tumours from 2 animals were sampled and a histological diagnosis of equine sarcoid was confirmed (Pathology Section of Onderstepoort Veterinary Institute). Four similar diagnoses in the GNR were made later in 1996.

The aim of this study was to document the outbreak of equine sarcoid in the GNR for the period 1995 to 2003. During this period the CMZ population varied between 50 and 75 animals (average population size was 69) and 39 cases of CMZ with sarcoid were observed.

\section{MATERIALS AND METHODS}

\section{Main study area - Gariep Nature Reserve}

The GNR is situated in the southern part of the Free State Province of South Africa along the northern shore of the Gariep Dam. The GNR (6000 ha) consists of steep flat-topped hills and undulating plains. The geological formations consist mainly of the Beaufort group sandstone and shale, which is shaped by dolerite dykes and sills. Average annual rainfall is $350 \mathrm{~mm}$ (range $150-500 \mathrm{~mm}$ ) and the vegetation is classified as eastern mixed Nama Karoo ${ }^{10}$. Temperatures vary from a minimum of $-10{ }^{\circ} \mathrm{C}$ in winter to a maximum of $42{ }^{\circ} \mathrm{C}$ in summer. The northern side of the GNR, which is game-fenced, borders commercial farmland with sheep, goats, cattle and horses.

\section{Animals and population dynamics}

According to the ecological management plan of the GNR, the CMZ population is managed to keep the population at between 50 and 70 animals. Excess animals are removed by live capture when the number approaches 70 animals. In 2002, a detailed investigation was conducted on the population. The 70 animals in the population were grouped into 16 herds, of which 11 were breeding herds with an average of 5 zebras and constituting $71 \%$ of the population total. The rest consisted of bachelor groups with an average of 4 zebras per group, making up the remaining $29 \%$ of the population. The male:female ratio was $1.2: 1$ and $70 \%$ of the animals were 3 years and older. No reliable data are available on contact rates between herds. Young males and females leave the breeding herd when they reach sexual maturity. Males join bachelor herds. Females are either gathered by a male from a bachelor herd to form a new breeding herd or they join other newly formed breeding herds. Herds sometimes mix if forced by some source of perceived danger (like chasing them for capture purposes) when they use the same escape route. Once left undisturbed they separate again.

Sarcoid-affected CMZ that had survived up to the end of 2003 were moved to a separately fenced area in the GNR in order to decrease the contact rate between affected and non-affected animals.

\section{Hands-off field observations}

The CMZ population at GNR was examined annually using standard $\times 10$ field binoculars. Where it was not possible to get close to the animals, a spotting scope ( $\times 20$ magnification) was used. An attempt 
was made to examine both sides of each animal in every herd. During these visual observations, the following were recorded on affected zebras: presence, number, estimated size (diameter) and location of tumour-like skin lesions as well as age and sex and the total number of individuals in the herd.

From field observations, the following epidemiological parameters were calculated:

- Number of affected animals in the population (prevalence). Animals which recovered after treatment trials were regarded as positive for the sake of prevalence calculations.

- Population at risk, which includes all animals in the population visually free of sarcoids.

- Adjusted population size (actual population number $-50 \%$ of live removals of healthy animals $+50 \%$ of births $-50 \%$ of natural mortalities not related to sarcoids) for a 12-month period.

- Number of new sarcoid cases in adjusted population at risk for the previous 12 month period (adjusted incidence)

- Age classes of affected animals.

- Sexes of affected animals.

- Herd structure of the population (2002 only).

- Age class composition of the population (2002 only).

- Male:female ratio in the population (2002 only).

\section{Hands-on examination}

Immobilisation for examination. Excluding 1999, all affected animals found during the annual capture period were immobilised using a Paxarms (Paxarms SA) remote injection rifle and darting from a helicopter. The herds were screened from the helicopter in a systematic manner. While flying at a low level every animal in each herd was observed visually from the sides and back for any cutaneous growths. Every effort was made to capture all affected animals (including recovered) in each herd for evaluation. Only 1 animal was immobilised at a time. After evaluation on the ground the animal was revived before continuing with the same herd.

Chemical immobilisation was performed using $24-26 \mu \mathrm{g} / \mathrm{kg}$ etorphine hydrochloride (M99, $9.8 \mathrm{mg} / \mathrm{ml}$, Novartis $\mathrm{AH}$ ) in combination with either $0.5 \mathrm{mg} / \mathrm{kg}$ azaperone (Azaperone, $100 \mathrm{mg} / \mathrm{ml}$, Kyron Laboratories) or $31-43 \mu \mathrm{g} / \mathrm{kg}$ detomidine hydrochloride (Domosedan, $10 \mathrm{mg} / \mathrm{ml}$, Novartis $\mathrm{AH}$ ). The etorphine $\mathrm{HCl}$ was reversed with an intravenous bolus of approximately $50 \mu \mathrm{g} / \mathrm{kg}$ diprenorphine hydrochloride (M5050, 12 mg/ml, Novartis $\mathrm{AH})$.
Observations. All affected animals were identified using individual ear notch combinations, plastic ear tags of different colours and shapes and photographs of each side using a Sony Cybershot 2.3 Megapixel digital camera. During hands-on examination, the following data were recorded: date of immobilisation; age ${ }^{19}$; sex; ear notch number; ear tag colour, sarcoid location and dimensions. The location of each lesion was noted as 1-location occurrence. Any treatments or other relevant information were also recorded. With the notch numbering system, animals were numbered sequentially from 1 onwards. A biopsy of each lesion was collected in $10 \%$ buffered formalin for histopathological confirmation of the diagnosis (results not presented here).

From the hands-on examinations and the field observation data, the following parameters/tendencies/features were noted:

- Predilection sites of the tumours on the body.

- Differences in predilection sites between male and female animals.

- Tumour multiplicity and the difference between male and female animals.

- Age at first onset (age determination was done using dental wear patterns ${ }^{19}$. The initial animals examined in 1996 were only classified into broader age groups and some cases were only visual observations from a distance, without exact ages being established).

- Change in sarcoid size.

\section{Sarcoid tumour size}

Owing to the fact that many sarcoids had irregular shapes, the length, width and height were measured and used to calculate a volume index $\left(V_{\mathrm{i}}\right)$.

The formula for the volume of a sphere was then used to calculate $V_{\mathrm{i}}$ using the average of length, width and height as the diameter. The formula used for the volume of a sphere is: $V=(\pi / 6) d^{3}$ where $V=$ volume and $d=$ diameter.

\section{Comparative investigations}

For the sake of comparison, the prevalence of sarcoid-resembling tumours was investigated in horses and Burchell's zebra (Equus quagga burchellii) in the Free State and CMZ in the Mountain Zebra National Park (MZNP) on a once-off basis during 2004. The Burchell's zebra were observed in 4 Free State provincial nature reserves, namely Tussen die Riviere, Maria Moroka, Koppies Dam and Sandveld. Burchell's zebra were examined using the same method as described for the CMZ at GNR (see 'Hands-off field observations' above). 'Tussen die Riviere'
Nature Reserve (23 000 ha) borders the GNR on the eastern side and has the same vegetation type $e^{10}$. Maria Moroka Nature Reserve (5500 ha) in the central Free State and Koppies Dam Nature Reserve (area 2457 ha) in the north both fall within the moist cool highveld grassland vegetation type ${ }^{10}$. Sandveld Nature Reserve (14000 ha), on the northwestern border of the Free State, consists of Kimberley thorny bushveld vegetation type ${ }^{10}$. The domestic horses investigated were on 2 commercial farms, both American saddle horse studs that border the GNR on the north. Cattle are also present on these farms and farmers were questioned about the presence of skin tumours on horses and warts in cattle. The CMZ $(n=300)$ in the MZNP in the Eastern Cape Province were also examined in 2004 for macroscopically visible sarcoid-resembling lesions. This population is possibly more out-bred than the GNR population. The MZNP (28 000 ha) has eastern mixed Nama Karoo (northern section) and southeastern mountain grassland (southern section) vegetation types ${ }^{10}$.

\section{RESULTS}

\section{Prevalence and incidence}

Basic data on the dynamics of the CMZ population at Gariep are presented in Table 1 . Because the population seldom remained static over a period of a year, the adjusted population size was calculated and used to determine the adjusted population at risk and adjusted incidence as well as the prevalence as presented in Table 2.

Monitoring of CMZ in GNR is not done at the intensity where all mortalities and births are recorded. The ruggedness of the terrain also makes the task very difficult. For this reason the net change was calculated from year to year based on the difference between births and natural mortalities.

The sarcoid prevalence in 4 populations of Burchell's zebra $(n=427)$ on other provincial nature reserves in the Free State was nil. With the exception of a prevalence of $0.9 \%$ in the CMZ at MZNP $(n=219)$, no sarcoid tumours were seen in either the Burchell's zebra populations, or the 2 horse populations $(n=80)$ adjoining the GNR.

\section{Age and sex distribution}

Distribution of sarcoid lesions according to age group and sex, based on first observation at GNR is presented in Table 3. Eleven ( 3 males and 8 females) of the 39 cases were not included in Table 3 on account of inaccurate age assessment (hands-off observations). In young CMZ, 
Table 1: Basic population dynamics data for Cape mountain zebra (CMZ) from Gariep Nature Reserve, 1996-2003.

\begin{tabular}{lccccc}
\hline Year & Population size & $\begin{array}{c}\text { Introductions } \\
\text { (sarcoid-free) }\end{array}$ & $\begin{array}{c}\text { Sarcoid-affected CMZ } \\
\text { removed from } \\
\text { population* }\end{array}$ & $\begin{array}{c}\text { Live removals of healthy } \\
\text { individuals }\end{array}$ & $\begin{array}{c}\text { Net change in population } \\
\text { to following year** }\end{array}$ \\
\hline 1996 & 64 & 0 & 6 & 5 & +17 \\
1997 & 71 & 5 & 1 & 0 & +6 \\
1998 & 58 & 0 & 2 & 5 & +8 \\
1999 & 62 & 0 & 0 & 0 & +9 \\
2000 & 65 & 0 & 2 & 0 & +7 \\
2001 & 72 & 0 & 2 & 0 & +12 \\
2002 & 77 & 0 & 7 & 20 & +3 \\
2003 & 82 & 0 & 19 & & +3 \\
\hline
\end{tabular}

*This column includes all mortalities (known and assumed), euthanased animals, and live removals to quarantine areas.

**Births minus natural mortalities during the year.

Table 2: Some population dynamics and epidemiological data for Cape mountain zebra from Gariep Nature Reserve, $1996-2003$.

\begin{tabular}{|c|c|c|c|c|c|c|}
\hline Year & Population size & $\begin{array}{l}\text { Adjusted population } \\
\text { size }\end{array}$ & $\begin{array}{l}\text { Adjusted population } \\
\text { at risk }\end{array}$ & $\begin{array}{l}\text { Number of new } \\
\text { cases }\end{array}$ & $\begin{array}{l}\text { Adjusted incidence } \\
(\%)\end{array}$ & $\begin{array}{c}\text { Prevalence at } \\
\text { end of period (\%) }\end{array}$ \\
\hline 1995-1996 & 64 & 70.5 & 64.5 & $6^{*}$ & & 9.4 \\
\hline 1996-1997 & 71 & 65.0 & 62.0 & 3 & 4.65 & 4.20 \\
\hline 1997-1998 & 58 & 61.0 & 59.0 & 0 & 0.00 & 3.40 \\
\hline 1998-1999 & 62 & 63.5 & 60.5 & 3 & 5.08 & 4.80 \\
\hline 1999-2000 & 65 & 69.5 & 62.5 & 4 & 6.61 & 10.80 \\
\hline 2000-2001 & 72 & 75.5 & 59.5 & 11 & 17.60 & 22.20 \\
\hline 2001-2002 & 77 & 83.0 & 64.0 & 5 & 8.40 & 24.70 \\
\hline 2002-2003 & 82 & 74.0 & 54.0 & 8 & 12.50 & 24.40 \\
\hline
\end{tabular}

*This number of cases could possibly have originated during a period of more than 12 months.

the first sarcoids were observed at 3 years of age. The difference in case numbers between age groups is, however, not statistically significant $\left(\chi^{2}=10.2, P<\right.$ $0.61)$.

Of the 39 sarcoid cases recorded from 1996 to 2003, $19(48.7 \%)$ were males and $20(51.3 \%)$ females. The difference in sex ratio of sarcoid-affected animals at different ages was found to be not statistically significant $\left(\chi^{2}=12, P<0.44\right.$. In 2002 the CMZ population $(n=82)$ consisted of $55.5 \%$ males $(n=40)$ and $44.5 \%$ females $(n=32)$. The remaining 5 foals and 5 subadults were not sexed. During the next year, 8 new cases were recorded, 3 of which were males and 5 females. Thus $7.5 \%$ of the males counted in 2002 developed sarcoid tumours, compared with $15.6 \%$ of females.

\section{Predilection sites and multiplicity}

Table 4 shows the anatomical distribution of all the sarcoids recorded during the study period. Several animals had more than 1 lesion in different locations. A total of 58 lesions were recorded on $39 \mathrm{CMZ}$; 29 on males and 29 on females.

Data on the number of tumours per animal are given in Table 5 . The majority of cases $(n=25)$ had only 1 lesion. No animal had more than 4 separate lesions on the body.

\section{Rate of sarcoid growth}

From 2001 to 2003, the average volume index of lesions on 1st observation $(n=$ 20) was $76 \mathrm{~cm}^{3}$. The epidemiological study coincided with a treatment trial where most of the affected animals $(n=22)$ were treated by 1 of 3 (autogenous vaccination, BCG treatment and surgery or a combination thereof) methods which resulted in a combined tumour regression rate of $77.5 \%$. The non-responders had an average annual increase in tumour size of $260 \%(n=5)$.

Table 3: Distribution of sarcoid cases $(n=28)$ according to age groups and sex in the Gariep Cape mountain zebra population, 1996-2003.

\begin{tabular}{lccccc}
\hline Sex & $<$ years & 3-6 years & 7-10 years & $>$ 10 years & Total \\
\hline Male & 0 & 6 & 5 & 5 & 16 \\
Female & 0 & 5 & 4 & 3 & 12 \\
Total & 0 & 11 & 9 & 8 & 28 \\
\hline
\end{tabular}

\section{DISCUSSION}

The outbreak of a disease in a threatened wildlife population is always a cause for concern. The approach to a disease outbreak in wildlife differs markedly from the approach in domestic animals. In wildlife, the objective is to correct the natural balance between the animal, the ecosystem and the disease agent to ensure the long-term survival of the population as opposed to the salvage of the individual animal for companionship, performance or reproductive purposes in domestic animals.

The outbreak was studied in order to determine the course of the disease in the population and the effect it would have on the dynamics of the population. Other aspects of the sarcoid outbreak were studied parallel to the epidemiology but fall outside the scope of this article.

Initial PCR and restriction enzyme digestion studies done on lesions from the Gariep CMZ resulted in BPV detection in all the samples ${ }^{28}$. Both BPV I and BPV II were found in the samples tested. This confirmed the assumption that the aetiology is similar to that in domestic equids.

BPV-DNA found in normal skin of horses affected with equine sarcoid ${ }^{4,11,27}$ could indicate viral latency. BPV was not found in the normal skin of horses without equine sarcoid ${ }^{4,18,26}$. Latency could 
Table 4: Location of sarcoids in male and female Cape mountain zebra, 1996-2003.

\begin{tabular}{|c|c|c|c|c|c|c|}
\hline Location & $\begin{array}{l}\text { Male lesions } \\
\quad(n=29)\end{array}$ & Male distrib \% & $\begin{array}{l}\text { Female lesions } \\
\quad(n=29)\end{array}$ & Female distrib \% & $M+F$ total & $M+F \%$ \\
\hline \multicolumn{7}{|l|}{ Head and neck } \\
\hline Ear & 1 & 3.45 & 4 & 13.79 & 5 & 8.62 \\
\hline Poll & 1 & 3.45 & 0 & 0.00 & 1 & 1.72 \\
\hline Cheek & 0 & 0.00 & 1 & 3.45 & 1 & 1.72 \\
\hline Lower jaw & 2 & 6.90 & 3 & 10.34 & 5 & 8.62 \\
\hline Throat & 1 & 3.45 & 1 & 3.45 & 2 & 3.45 \\
\hline Eye & 0 & 0.00 & 3 & 10.34 & 3 & 5.17 \\
\hline Neck & 1 & 3.45 & 0 & 0.00 & 1 & 1.72 \\
\hline Head and neck combined & 6 & 20.69 & 12 & 41.38 & 18 & 31.03 \\
\hline \multicolumn{7}{|l|}{ Trunk and legs } \\
\hline Chest and trunk & 3 & 10.34 & 4 & 13.79 & 7 & 12.07 \\
\hline Inguinal & 16 & 55.17 & 8 & 27.59 & 24 & 41.38 \\
\hline Perineal & 0 & 0.00 & 1 & 3.45 & 1 & 1.72 \\
\hline Distal limb & 0 & 0.00 & 1 & 3.45 & 1 & 1.72 \\
\hline Elbow medial & 0 & 0.00 & 2 & 6.90 & 2 & 3.45 \\
\hline Knee medial & 4 & 13.79 & 1 & 3.45 & 5 & 8.62 \\
\hline Trunk and legs combined & 23 & 79.31 & 17 & 58.62 & 40 & 68.97 \\
\hline Total & 29 & 1.00 & 29 & 1.00 & 58 & 2.00 \\
\hline
\end{tabular}

make the determination of incubation period difficult. Activation of latent papillomavirus genomes by chronic epidermal irritation has been documented in Mastomys natalensis ${ }^{24}$. It has also been noted that sarcoids in horses tend to occur in areas of skin damage ${ }^{9}$. It is possible that for BPV-DNA to be activated, some form of epidermal damage or irritation is necessary for the activation of transacting factors which bind to the regulatory regions causing expression of the viral genome which then leads to sarcoid development.

Incubation period was not determined in CMZ. In a case of experimental autotransmission between horses by topically inoculating scarified skin, the incubation period was 6 months while another study indicated that incubation period differed between homologous transmission (115 days) and autologous transmission (57 days) in transmission studies conducted ${ }^{16,29}$. Incubation periods of 5 and 10 months have been noted in 2 donkeys inoculated intradermally with a cell-free sarcoid extract from a horse ${ }^{21}$. It is extremely difficult to determine the incubation period in an extensive CMZ population, as lesions are only seen when they reach a certain size.

Transmission studies in horses have been relatively unsuccessful. Scarified skin seems to be more receptive to sarcoid development than sub-cutaneous or intradermal inoculations ${ }^{12,17,29}$. Although there are strong indications that flies are responsible for the spread of sarcoids from one site to another in an individual horse, it is uncertain whether insects are responsible for transmission between horses. Close association between animals was shown to be a risk factor in transmission of $\mathrm{BPV}^{22}$. As no viral genomes have been found in the blood of affected horses, spread via the bloodstream is not regarded as a method of spread ${ }^{9}$. No BPV-DNA was found in blood or normal skin of sarcoid-affected CMZ from GNR in a preliminary study (E van Dyk, Faculty of Veterinary Science, University of Pretoria, pers. comm., 2004).

The locations of sarcoid lesions in the Gariep CMZ population were predominantly in areas of thin skin and a sparse hair cover (Table 4) and correspond with observations in horses ${ }^{9}$. This may support the theory of an insect vector ${ }^{9}$, as these sites could be the preferred sites for a biting insect vector. It is, however, a hypothesis that will require further investigation. It is interesting to note that no sarcoid lesions were ever recorded in the 2 horse studs adjoining the GNR. Although the CMZ do not have direct contact with the horses or cattle on the adjoining farm land, they do sometimes graze within a few hundred metres from them. One of the farmers adjoining the GNR has seen isolated cases of warts on his cattle. It is possible that

this could have been the source of the papilloma virus for the initial infection of the CMZ. The fact that no horses developed sarcoid could be an indication that the Gariep CMZ are more susceptible to the development of equine sarcoid compared with the domestic horses occurring next to the reserve. Although the reason for this increased susceptibility has not been proven, speculation and initial genetic results suggest that inbreeding depression may be one of the factors responsible $^{23}$.

When the Gariep CMZ population was examined in 1996 after the first reports of 'skin growths' in late 1995, the prevalence of affected animals was $9.4 \%$. After all the visibly affected $\mathrm{CMZ}$ were removed from the population in 1996, the prevalence was $4.6 \%$ in 1997, after which it increased gradually to about $24 \%$ in 2002/03. Owing to the rugged terrain and level of monitoring on the ground, few carcasses were found. Sarcoid-affected animals not found on follow-up investigations were presumed to be dead. During 2003 all affected animals $(n=16)$ were moved to another camp in order to decrease the contact rate and possible transmission between affected and non-affected. From

Table 5: Number of sarcoid tumours per animal in the Gariep Cape mountain zebra $(n=39)$.

\begin{tabular}{lrrrr}
\hline Number of tumours & Male & Female & Total & Total \% \\
\hline 1 & 10 & 15 & 25 & 64.10 \\
2 & 6 & 4 & 10 & 25.64 \\
3 & 2 & 1 & 3 & 7.69 \\
4 & 0 & 1 & 39 & 2.56 \\
Total & 18 & 21 & 39 \\
\hline
\end{tabular}


1996 to 2003, a total of 86 animals were removed from the population. This included mortalities and live removals. Thirty-nine were sarcoid affected and 47 were unaffected. The annual population number varied from 64 to 82 zebras. Mortality due to other causes is not included in these figures. The average annual recruitment was $17.8 \%$ for the study period. If all sarcoid-affected individuals had been removed as soon as they were seen, the average annual productivity of the population would have only been $9.8 \%$. If natural mortality is deducted, the population comes very close to being stagnant. Although no sarcoid cases were found during the once-off investigation at Maria Moroka NR, it is known that from 1992 to 2004, 1 case of equine sarcoid in a Burchell's zebra was diagnosed in this reserve. The $24 \%$ sarcoid prevalence in the Gariep $\mathrm{CMZ}$ population is therefore highly significant. The same would apply to the high prevalence of equine sarcoid (close to $50 \%$ ) reported for the $\mathrm{CMZ}$ population from the Bontebok National Park (D Zimmerman, South African National Parks Board, pers. comm., 2003).

After the initial removal of all visibly affected zebra in 1996, incidence increased from $4.6 \%$ in 1997 to $17.6 \%$ in 2001 , after which it decreased again to $8.4 \%$ and $12.5 \%$ in 2002 and 2003, respectively. As prevalence increases, the contact rate between susceptible and affected zebra would increase, thereby explaining the annual increase in incidence up to 2001. The sudden drop in incidence after 2001 could have been as a result of a decrease in the number of susceptible individuals in the population but could also be as a result of other factors. Most of the susceptible animals could have been infected in previous years while susceptible offspring provided the majority of susceptible individuals.

From studies in horses it can be concluded that not all individuals are susceptible to the development of sarcoid. Until more is known about the genetics of the disease, it is not possible to predict whether an individual is susceptible to sarcoid development ${ }^{9}$. Initial examination of the heterozygosity status of the Gariep CMZ population using genetic microsatellite studies $(n=$ 5) in 1995 indicated a significant level of inbreeding (E H Harley, University of Cape Town Medical School, pers. comm., 1995). During 2003, 17 sarcoid-affected CMZ from Gariep were sampled in order to conduct further comparative microsatellite investigations. This was compared with CMZ populations at the Karoo National Park (KNP), Karoo Nature
Reserve (KNR), Bontebok National Park (BNP) and a population of Hartmann's mountain zebra (Equus zebra hartmannae) from Namibia ${ }^{23}$. The population from $\mathrm{KNP}$ is one of the 2 largest populations $(n \approx 300)$ of CMZ in South Africa. The Hartmann's mountain zebra (HMZ) meta-population is currently estimated at 20 000-30 000 animals $^{15}$. KNP, KNR, GNR and BNP populations were all seeded from the population at the Mountain Zebra National Park. Mean heterozygosity values were lowest for the tumouraffected populations (0.386) compared with tumour-free populations (0.427) and HMZ (0.609). Mountain zebra from GNR exhibited lower heterozygosity values than BNP. CMZ from Karoo National Park (KNP) also had low levels of polymorphism and exclusion probability values. However, higher heterozygosity values were recorded when compared with BNP and GNR. Gene diversity values were lowest for tumour-affected populations $(39.5 \%)$ and highest for Hartmann's mountain zebra (52.3\%). The overall value for Nei's population differentiation parameter (Gst'), also an indicator of heterozygote deficit, illustrated that tumour-affected CMZ populations have a greater degree of differentiation (0.156) and deficit of heterozygote than tumourfree CMZ (0.082) and Hartmann's zebras (0.08). The mean number of alleles was lowest in the diseased populations (BNP and GNR; 2.22), with GNR having the lowest polymorphic information content. The diseased population had lower comparative allele richness values (2.33 vs 2.64) than the tumour-free animals and the HMZ (5.896). The level of relatedness was high between all CMZ populations, with the diseased population showing comparatively higher values than tumour-free animals (0.29 vs 0.17). This investigation revealed that the 2 diseased populations have the lowest genetic variation of the 4 mountain zebra populations investigated and are representative of most extant CMZ populations. Apart from being highly inbred, these populations have a high level of genetic sub-structuring. It could thus be demonstrated that $\mathrm{CMZ}$, after surviving the historically documented genetic bottleneck, have very little genetic variation left $\mathrm{ft}^{23}$.

It is generally suggested by various studies that multigenerational consanguinity often significantly affects birth weight, survival, reproduction, resistance to disease, predation and environmental stress $^{23}$. It could therefore be argued that the high sarcoid prevalence in the Gariep CMZ population could be influenced by the degree of inbreeding. The fact that 2 neighbouring domestic horse popula- tions (average size of the 2 groups during the study period was 80 horses) did not have any sarcoid cases during the study period also indicates that the Gariep CMZ population has an abnormally high susceptibility to equine sarcoid. Out of 427 Burchell's zebra examined on other Provincial Nature Reserves in the Free State, no suspect sarcoid lesions were seen (although 1 case has been confirmed as equine sarcoid during the period 1992-2004 in Burchell's zebra from these populations). Out of $72 \%$ of a population of CMZ $(n=305)$ which was examined visually in 2004 at the Mountain Zebra National Park, only $0.91 \%$ of the animals had lesions resembling equine sarcoid. This could, however, be as a result of a relatively recent introduction of BPV with the potential to develop into a significant outbreak with a high incidence. The only other CMZ population previously known to be affected by equine sarcoid is the population at the Bontebok National Park (BNP) which had a prevalence of $18.75 \%$ in 2000 and $53 \%$ in $2002^{30}$. The high prevalence of equine sarcoid in inbred populations of CMZ compared with that in larger, more outbred CMZ populations, Burchell's zebra or horses is clearly significant.

No conclusive breed predilection was found amongst horses in a study in Queensland ${ }^{13}$. In another study it was, however, concluded that there seemed to be some breed predilection in horses regarding sarcoid prevalence ${ }^{12}$. Quarter horses were found to have almost twice the risk of developing sarcoids compared to Thoroughbreds, while Standardbreds had a much lower risk relative to all other breeds ${ }^{2}$. The association between certain equine leukocyte antigen (ELA) haplotypes and sarcoid susceptibility and expression is the strongest association between MHC genes and disease in any species. The presence of the A5 haplotype has been associated with early onset $(2.9 \pm 1.5$ years $)$ of equine sarcoid while the W13 haplotype has been associated with increased risk of recurrence ${ }^{12,20}$. The precise MHC genes responsible for sarcoid susceptibility have not yet been identified $^{12}$. Familial tendencies towards the development of equine sarcoid have been described in $80 \%$ of horses from a highly inbred lineage ${ }^{8}$. All these reports from domestic equids support the notion that the high incidence of equine sarcoid in inbred CMZ populations may be as a result of inbreeding depression.

It has been found in horses that different age groups were almost equally susceptible to sarcoids, with the 1-6-year-old group the most common age at which sarcoids were diagnosed ${ }^{13}$. A similar trend 
was observed in the Gariep outbreak. The earliest age at which equine sarcoid has been found in horses is in yearlings ${ }^{12}$. In the Gariep outbreak, no animals younger than 3 years were seen with sarcoid. They could, however, have been infected as yearlings and with an incubation period of a few months, sarcoids would start to develop during the 2 nd year and would only be large enough to be seen the following year when they were 3 years old. At the Bontebok National Park 41.6\% of the 12 sarcoid cases in CMZ were first seen when the animals were $2-2.5$ years old compared with $10.25 \%$ in the Gariep population that were 3 years old. The variation in the different age classes affected (Table 3 ) is not statistically significant $\left.\chi^{2}=10.2, P<0.61\right)$.

As this is a case study, the small number of samples often hampered statistical analysis.

The sex ratio of sarcoid-affected cases in the Gariep population is close to $1: 1$. Although the population was male biased during 2002 ( $55.5 \%$ male: $45.5 \%$ female) it does not necessarily mean that this was the case during the whole study period. Unfortunately the male:female ratio of the Gariep population was not determined annually. During game reduction operations, more female than male animals are usually removed. This could have resulted in the male biased population in Gariep as was observed in 2002. Of the 12 sarcoid cases from the BNP population, $33.3 \%$ were males and $66.7 \%$ females. This, however, is very close to the male:female ratio in BNP. No sexual predilection was found in a survey on equine sarcoid in domestic horses in Queensland ${ }^{13}$.

Growth rate of sarcoids has not been described in domestic horses. The only control animal (no treatments received) in the GNR study died before recapture the following year so it is impossible to determine what the natural growth rate of the tumour would have been. It is important to note that even the biopsy procedure could trigger an immune response which might influence the growth rate of the sarcoid. Thus if sarcoid growth rate is to be determined, biopsies for confirmation of histological diagnosis should only be taken once the control trial has been finalised.

Although sarcoids can occur on any part of the body, the location of sarcoid tumours on the body of horses varies between clinics and different locations where studies have been conducted ${ }^{5,12}$. In a South African survey, it was found that in horses, $46.5 \%$ of sarcoids occurred on the head, $6.8 \%$ on the abdomen, $17.2 \%$ in the inguinal area, $8.6 \%$ on the chest, $19 \%$ on the limbs and $1.7 \%$ on the neck. Of the sarcoids located on the head, $41 \%$ were situated on the ears, $15 \%$ on the eyelids and $44 \%$ on the rest of the head. Of the abdominal sarcoids $71.4 \%$ were located in the inguinal area ${ }^{3}$. In a Queensland survey it was found that $40 \%$ of sarcoids occurred on the lower leg, $18 \%$ on the upper leg, $1 \%$ on the abdomen, $15 \%$ on the chest and trunk, $25 \%$ on the head and neck and $1 \%$ on the genitalia. It was concluded that sarcoids are commonly found on areas on the horse most susceptible to trauma ${ }^{13}$ and areas with thin skin, limited hair cover and areas with a tendency to sweat ${ }^{9}$. In the United Kingdom, sarcoids are rare on the upper trunk, back and neck. When they do occur at these sites, it is usually as a result of traumatic damage to the skin. In the UK, lesions on the distal limb are also rare and usually resulted after skin damage (either new or old lesions). Predilection sites in horses summarised from 5 studies representing 662 sarcoids were: $45.8 \%$ on the limbs, $31.6 \%$ on the head and neck, $8.8 \%$ chest and trunk, $6 \%$ abdomen and flanks, $3.6 \%$ preputial, $0.3 \%$ at castration wounds and $3.6 \%$ at other sites ${ }^{25}$. Sites predisposed to trauma or contact with other sarcoids also show a higher tendency for sarcoid occurrence. In the Gariep CMZ, 31 \% of lesions occurred on the head and neck while $41.4 \%$ of lesions were found in the inguinal region (Table 4). There is, however, an interesting difference between males and females. In males the inguinal lesions accounted for $55.2 \%$ of lesions while the head and neck accounted for $20.7 \%$ and the area medial to the stifle accounted for $13.8 \%$. Together these 3 locations account for $89.7 \%$ of sarcoid lesions $(n=29)$ in males. In females, $27.6 \%$ of lesions occur in the inguinal area, $41.4 \%$ on the head and neck and $3.5 \%$ on the area medial to the stifle joint. Compared with females, the male zebra has a larger surface area of thin hairless skin in the inguinal area which could make it relatively more prone to BPV transmission from biting insects in this area. Lesions on the head are also usually on areas with sparse hair cover (eyelids, ears and the soft thinskinned area around the muzzle). In the BNP population, $33.3 \%$ of lesions are located in the region of the axilla, $25.6 \%$ of lesions on the trunk, usually on the ventral abdomen, and $10.3 \%$, respectively, on the head, chest and shoulders and the area medial to the stifle joint ${ }^{30}$. Although there is no scientific evidence at this stage, it is possible that different vectors with different biting site preferences could be responsible for the difference in sarcoid locations between horses and CMZ, as well as for differences be- tween different sarcoid-affected CMZ populations. It is possible that habitat can play a role where injuries can be caused by moving through certain plants, which would attract vectors and could be an entrance point for BPV. Apart from chance (small sample size), there is currently no other logical explanation for the differences in lesion locations between different equids.

In the Gariep CMZ the majority of affected animals had a single lesion and none of the animals had more than 4 lesions. Similar results were seen in a survey in Queensland where 22,2 \% of horses affected by sarcoid had multiple lesions of which approximately $63 \%$ had 2 lesions, $25.7 \%$ had 3 lesions, $2.8 \%$ had 4 lesions, $5.7 \%$ had 5 lesions and $2.8 \%$ had more than 5 lesions $^{13}$. In the BNP outbreak, $7(53.9 \%)$ animals had a single lesion, 2 animals $(15.4 \%)$ had 2 lesions, and 4 animals each had 3, 5, 7 and 13 lesions, respectively ${ }^{30}$. The average number of lesions per horse in continental Europe is about 3 compared with 25-30 lesions per horse in the UK $\mathrm{UK}^{9}$. Efficiency of vector transmission, climate, contact rate of vectors with infective material, host susceptibility, viral virulence, virus strain and numerous other variables could be responsible for differences in the number of lesions per individual.

Equine sarcoid has for many years been a difficult clinical entity to deal with in horses. In a free-ranging wildlife population, where minimal human interference is the ideal, it presents an even more difficult problem to deal with. Because genetic susceptibility to equine sarcoid is a strong possibility, the disease in CMZ should be researched most intensively in the cytogenetic field. The danger of offspring inheriting susceptibility to equine sarcoid can at the moment only be addressed by removing affected individuals from the gene pool. The reverse side of the problem is that the CMZ meta-population is already inbred ${ }^{23}$ and the removal of individuals only exacerbates the problem. Apparent unwillingness of certain conservation agencies to provide unrelated male breeding stock for introduction to inbred populations is also a problem. Ideally if one could identify female individuals which are genetically susceptible to equine sarcoid and remove them to an isolated breeding area where they are cross-bred with non-susceptible males, the population could over time be bred to be sarcoid resistant with improved genetic variability. It was found at the GNR and at the $\mathrm{BNP}^{30}$ that treatment with autogenous vaccine, surgery and BCG (BCG vaccine SSI, Statens Serum Institute, Denmark), caused tumour regression in 
the majority of sarcoid-affected CMZ, which makes it possible to salvage affected individual for out-breeding projects. Not being able to identify genetically resistant and susceptible CMZ is at this stage the most crucial shortcoming in managing this disease in the endangered Cape mountain zebra.

\section{ACKNOWLEDGEMENTS}

Sincere appreciation is expressed to the Free State Department of Tourism, Environment and Economic Affairs for allowing this study to be conducted and all the personnel who assisted with different aspects of the study. We would like to thank Prof. Alan Guthrie, Director of the Equine Research Centre, University of Pretoria, for assistance in the project.

\section{REFERENCES}

1. Angelos J A, Marti E, Lazary S, Carmichael L E 1991 Characterization of BPV-like DNA in equine sarcoids. Archives of Virology 119: 95-109

2. Angelos J, Oppenheim Y, Rebhun W Mohammed H, Antczak D F 1988 Evaluation of breed as a risk factor for sarcoid and uveitis in horses. Animal Genetics 19 417-425

3. Bastianello S S 1983 A survey on neoplasia in domestic species over a 40-year period from 1935 to 1974 in the Republic of South Africa IV. Tumours occurring in equidae. Onderstepoort Journal of Veterinary Research 50: 91-96

4. Carr E A, Théon A P, Madewell B R, Griffey S M, Hitchcock M E 2001 Bovine papillomavirus DNA in neoplastic and non-neoplastic tissues obtained from horses with and without sarcoids in the western United States. American Journal of Veterinary Research 62: 1212-1217

5. Foy J M, Rashmir-Raven A M, Brashier M K 2002 Common equine skin tumours. Equine Compendium 24: 242-253

6. Friedman Y, Daly B (eds) 2004 Red Data Book of the mammals of South Africa: a conservation assessment: GBSC South Africa. Conservation Breeding Specialist Group (SSC/IUCN), Endangered Wildlife Trust, South Africa

7. Jackson C 1936 The incidence and pathology of tumours of domestic animals in South Africa, a case study of the Onderstepoort collection of neoplasms with special reference to their histopathology. Onderstepoort Journal of Veterinary Research 6: 378-249

8. James V S 1968 A family tendency to equine sarcoids. Southwest Veterinarian 6: 378-385.

9. Knottenbelt D C 2001 Facts you need to know about sarcoids. Proceedings of the 33rd Annual Congress of the Equine Practitioners Group of the SAVA, 33-42

10. Low A B, Rebelo A G 1996 Vegetation of South Africa, Lesotho and Swaziland. Department of Environmental Affairs and Tourism, Pretoria

11. Martens A., De Moor A., Ducatelle R. 2001 PCR detection of bovine papillomavirus DNA in superficial swabs and scrapings from equine sarcoids. The Veterinary Journal 161: 280-286

12. Marti E, Lazary S, Antczak D F, Gerber H 1993 Report of the first international workshop on equine sarcoid. Equine Veterinary Journal 25: 397-407

13. Miller R I, Campbell R S F 1982 A Survey of granlomatous and neoplastic diseases of equine skin in north Queensland. Australian Veterinary Journal 59: 33-37

14. Moehlman P D 2002 Equids: Zebras, asses and horses. IUCN - The world conservation union status survey and conservation action plan: 94-123

15. Novellie $P$, Lindeque $M$, Lindeque P, Lloyd P, Koen J 2002 Status and Action Plan for the Mountain Zebra (Equus zebra). IUCN/SSC Equid Specialist Group 28: 8-42

16. Olson C Jr. 1948 Equine sarcoid: a cutaneous neoplasm, American Journal of Veterinary Research 9: 333-341

17. Olson C Jr., Cook R H 1951 Cutaneous sarcoma-like lesions of the horse caused by the agent of bovine papilloma. Proceecings of the Society for Experimental Biology and Medicine 77: 281-284

18. Otten N, Von Tschamer C, Lazary S, Antczak D F, Gerber H 1993 DNA of bovine papillomavirus type 1 and 2 in equine sarcoids: PCR detection and direct sequencing. Archives of Virology 132: 121-131
19. Penzhorn B L 1982 Age determination in cape mountain zebras Equus zebra zebra in the Mountain Zebra National Park. Koedoe 25: 89-102

20. Piscopo S E 1999 The complexities of sarcoid tumors. Equine Practice 21(8): 14-18

21. Ragland W L, Keown G H, Spencer G R 1970 Equine sarcoid. Equine Veterinary Journal 2: 2-11

22. Reid S W J, Smith K T, Jarrett W F H 1994 Detection, cloning and characterisation of papillomaviral DNA present in sarcoid tumours of Equus asinus. Veterinary Record 135: 430-432

23. Sasidharan S P 2004 Comparative genetics of selected Southern African Mountain Zebra (Equus zebra zebra and Equus zebra hartmannae) populations. MSc dissertation, University of Pretoria

24. Siegsmund M, Wayss K, Amtmann E 1991 Activation of latent papillomavirus genomes by chronic mechanical irritation. Journal of General Virology 72: 2787-2789

25. Sullins K E, Roberts S M, Lavach J D, Severin G A 1986 Equine sarcoid. Equine Practice - Dermatology 8(4): 21-27

26. Teifke J P, Hardt M, Weiss E 1994 Detection of bovine papillomavirus DNA in formalin-fixed and paraffin-embedded equine sarcoids by polymerase chain reaction and non-radioactive in situ hybridization. European Journal of Veterinary Pathology 1: 5-10

27. Trenfield K, Spradbrow P B, Vanselow B 1985 Sequences of papillomavirus DNA in equine sarcoids. Equine Veterinary Journal 17: 449-452

28. Van Dyk E, Bosman A-M, Venter E H, Nel P J, Zimmerman D 2004 Detection of bovine papillomavirus DNA in sarcoids in the Cape mountain zebra (Equus zebra zebra) In: Faculty Day Proceedings, Onderstepoort; 2004 Sep 16; Onderstepoort

29. Voss J L 1969 Transmission of equine sarcoid. American Journal of Veterinary Research 30: 183-191

30. Zimmerman D 2004 Summary report and draft action plan for the equine sarcoid problem in cape mountain zebra at Bontebok NP. Internal Report, November 2004, Veterinary Wildlife Unit, South African National Parks Board, Kimberley 